\title{
144. APORTACIONES AL CONOCIMIENTO DE LA FLORA Y VEGETACIÓN DEL CENTRO-OCCIDENTE IBÉRICO (CW DE ESPAÑA Y NE DE PORTUGAL)
}

\author{
Sonia BERNARDOS, Anabela AMADO, \\ Carlos AGUIAR, Antonio L. CRESPÍ, Adriano CASTRO y Francisco AMICH
}

\begin{abstract}
Flora and Vegetation of central-western Iberian Peninsula (CW of Spain and NE of Portugal)
\end{abstract}
Palabras clave. Flora, vegetación, CW ibérico, Lusitano Duriense, Toledano Tagano, corología.

Key words. Flora, vegetation, central-western Iberia, Lusitan Duriensean, Toledan Taganean, chorology.

Continuando con nuestros estudios sobre la Flora y Vegetación en el centro occidente ibérico, y en particular en la Cuenca del Duero (p.e. Amado et al. in Aguiar et al., 2003; Bernardos et al., 2003; Crespí et al., 2003; Bernardos et al., 2004), presentamos ahora diversos resultados de índole corológica, taxonómica y fitosociológica obtenidos en estos territorios lusitano durienses, así como en el sector biogeográfico Toledano Tagano. Aportamos nuevas citas de diversas especies en la zona estudiada, ampliamos asimismo el área de distribución de varios táxones y, por último, discutimos el encuadre sintaxonómico de algunas especies relevantes.

Para la nomenclatura de los táxones hemos seguido Flora iberica (Castroviejo et al. 1986-1997, 2003; Muñoz Garmendia \& Navarro, 1998; Paiva et al., 2001, Talavera et al., 1999-2000) y Flora Europaea (Tutin et al., 1964-1980), excepto para Crambe hispanica subsp. glabrata (DC.) Cout. in Fl. Portugal: 272 (1913), Galium glaucum subsp. australe Franco in Nova Fl. Portugal 2: 79.1984 (= Galium teres Merino in Fl. Galicia 2: 286. 1906), Scrophularia valdesii Ortega Olivencia \& Devesa in Candollea 46: 115. 1991 (= S. grandiflora subsp. reuteri sensu Amich, Anales Jard. Bot. Madrid 36: 295. 1980, non Daveau (1892)), Sideritis danielii Rivera \& Obón in Phaner. Monog. Tomus XXI: 413. 1994 (= S. bubani sensu Franco in Nova F1. Portugal 2: 150, non Font Quer (1920)), Silene boryi subsp. duriensis (Samp.) Coutinho in Fl. Portugal.: 220. 1913 y Trigonella polyceratia subsp. amandiana (Samp.) Amich \& J. Sánchez in Stud. Bot. Univ. Salamanca 2: 129. 1983.

El material colectado se encuentra depositado en alguno de los siguientes Herbarios: Universidad de Salamanca (SALA), Universidade de Trás-os-Montes e Alto Douro (HVR) y Escola Superior Agrária de Bragança (BRESA), siglas aún no recogidas en el Index Herbariorum). En ocasiones, cuando las poblaciones eran escasas, no se recogió material, y se cita como testimonio una diapositiva, depositada en la colección de uno de los autores (F. Amich).

Un GPS Garmin e-map ha sido utilizado para la correcta localización de los taxa en cuadrículas UTM de 1 x $1 \mathrm{~km}$.

La nomenclatura sintaxonómica seguida por los autores es la propuesta por RivasMartínez et al. (2001, 2002). Desde el punto de vista biogeográfico seguimos los criterios de Rivas-Martínez et al. (1999, 2002).

Lycopodiella inundata (L.) J. Holub SPA, SALAMANCA: Herguijuela de la 
Sierra, Valle de Belén, 820 m, 29TQE4580, 30.5.2003, Amich \& Bernardos, Diapositiva $n^{\circ}$. 5323.

Segunda localidad salmantina de esta relevante especie, a añadir a la de Rico (1980). La hemos encontrado ahora en la vertiente sur de la sierra de Francia, muy cerca de la vecina provincia de Cáceres, en territorios pertenecientes a la cuenca del río Alagón, en el sector biogeográfico Toledano Tagano.

Vive en pequeños emplazamientos higroturbosos referibles a la asociación Eleocharito multicaulis-Rynchosporetum albae, siendo muy escasa en los mismos.

Silene boryi Boiss. subsp. duriensis (Samp.) Cout.

PO, BEIRA ALTA: Casais do Douro, Bateiras, 29TPF25, 15.5.1941, Pedro 1268, HVR 1276; Em frente à Régua, margem do Douro, 29TPF05, 6.7.1941, Pedro \& Lopes 2204, HVR 1275; Entre o Pocinho e a foz do Côa, entre a Quinta do Meão e a Quinta do Reguengo, 29TPF55, 9.6.1942, Myre \& Barbosa 4356, HVR 1273; Entre Castelo Melhor e a foz do Côa, 29TPF55, 15.6.1942, Barbosa \& Myre 4280, HVR 1277.

PO, TRÁS-OS-MONTES: Vila Real, Régua, Foz do Corgo, $180 \mathrm{~m}, 29 \mathrm{TPF} 05,25.4 .1990$, Coelho, HVR 12589; Bemposta, barragem da Bemposta, 360 m, 29TQF1174, 15.5.2003, Amado \& Amich, SALA 108460.

Confirmamos ahora la presencia de este taxon en Trás-os-Montes. A pesar del tratamiento taxonómico de Talavera (1990), las plantas que viven en las márgenes del Duero, tanto españolas como portuguesas, presentan un conjunto de caracteres morfológicos diagnósticos -que el mismo monógrafo destaca-, así como un areal propio -sector biogeográfico Lusitano Duriense, que creemos justifican claramente su separación con este rango de otras poblaciones ibéricas.

Amado et al. (in Aguiar et al., 2003) describen para las márgenes del río Duero, en zonas administrativamente portuguesas, una original comunidad permanente dominada por Aphyllanthes monspeliensis y Coronilla minima subsp. lotoides, y en la que se integran algunos de los pocos elementos endémicos de este sector biogeográfico Lusitano Duriense, tales como Silene boryi subsp. duriensis, así como algunas de las más relevantes disyunciones conocidas para el mismo, como Globularia vulgaris, Polygala microphylla, Scorzonera hispanica subsp. crispatula y Sideritis danielii. Los autores de la asociación presentan un total de 8 inventarios, todos ellos correspondientes a la misma localidad (Trás-os-Montes: Bemposta, barragem da Bemposta), aunque indican que han detectado esta comunidad en Miranda do Douro y en las márgenes españolas del río Duero frente a la presa de Bemposta. Por ello, y ante la escasez de datos sobre la distribución geográfica de esta particular comunidad, hemos estudiado todo el tramo fronterizo del río Duero, entre el Embalse de Castro (Zamora) y el Muelle de Vegaterrón (Salamanca), para conocer la composición de la misma y su sincorología. Como resultado presentamos un total de 6 inventarios (tab. 1) repartidos de manera uniforme a lo largo de este tramo fronterizo, que confirman la presencia de Sileno-Aphyllanthetum en varias localidades españolas. En cuanto a su composición, parece bastante constante a lo largo de todo el territorio estudiado (tab. 1), con sólo muy pequeñas variaciones en su combinación florística; en las poblaciones portuguesas de Bemposta cuenta con elementos basófilos del occidente mediterráneo, como Fumana ericifolia (Amado \& Aguiar, 2004), que no encontramos en las poblaciones españolas situadas más al sur; por el contrario, en alguna de estas últimas se integran plantas como Hippocrepis comosa, sufrútice no conocido hasta el momento de Trás-os-Montes ni de Portugal (Talavera \& Domínguez, 2000).

A pesar de la presencia de algunos táxones típicos de las comunidades de Rumici induratiDianthion lusitani presentes en estos territorios, tales como Galium glaucum subsp. australe o Petrorhagia saxifraga, predominan sin duda los elementos característicos de Rosmarinetalia, tales como Aphyllanthes, Coronilla, Fumana, Globularia, Hippocrepis, Scorzonera, etc. (Rivas-Martínez et al., 2002), por lo que, de acuerdo con los autores del sintaxon, llevamos a ese orden las comunidades estudiadas.

Dentro de este orden, la adscripción parece 
clara a la alianza Sideritido-Salvion y, en concreto, a la subalianza Xero-Aphyllanthenion; al tratarse de unas comunidades características de la subprovincia Castellana (provincia Mediterránea Ibérica Central), que alcanzan de manera empobrecida, a través de la cuenca superior y media del Duero, estos territorios lusitano durienses de la subprovincia Carpetano Leonesa (provincia Mediterránea Ibérica Occidental).

Sileno duriensis-Aphyllanthetum monspeliensis es, por lo que conocemos hasta ahora, un interesante sinendemismo del sector corológico Lusitano Duriense.

\section{Cleome violacea $\mathrm{L}$.}

PO, BEIRA ALTA: São João da Pesqueira, Vale da Figueira, 29TPF3956, 18.5.2001, Crespí et al. 187, HVR 12460.

PO, TRÁS-OS-MONTES: Bragança, Quintanilha, Portelas, caminho florestal, ca. 700 m, 29TPG92, 6.7.1993, C. Aguiar 2205, BRESA 2608; Macedo de Cavaleiros, a montante da ponte de Remondes, acima de um caminho que desce para Lagoa, 29TPF 88, 18.5.1997, Koe \& Castro 1697, HVR 9531; Mogadouro, a jusante da ponte de Remondes, na margem direita do rio Sabor, 29TPF88, 13.6.1997, Sequeira \& Castro 3113, HVR 9532.

SPA, SALAMANCA: Cepeda, riberas del río Francia, 615 m, 29TQE5182, 9.7.2003, Amich \& Bernardos, SALA 108458; Cepeda, riberas del río Alagón, 645 m, 29TQE5385, 9.7.2003, Amich \& Bernardos, SALA 108459.

Nuevas localidades salmantinas, correspondientes en este caso a la cuenca del Alagón, a añadir a las conocidas en Las Arribes del Duero, en el occidente provincial (Amich, 1980) y en la Sierra de Béjar (Amich, Rico \& Sánchez, 30.6.1981, MA 243822), citas que fueron posteriormente recogidas en los mapas de distribución del taxon de Álvarez et al. (1996) y Álvarez Maestre \& Santos Esteban (1996). Se trata de una planta muy escasa en el centro occidente ibérico, tanto en España como en Portugal, de donde aportamos asimismo nuevas localidades altobeirenses y trasosmontanas.

Crambe hispanica L. subsp. glabrata (DC.) Cout. PO, BEIRA ALTA: Figueira de Castelo
Rodrigo, Almofala, tributário do rio Águeda, 29TPF8226, 24.4.1994, Koe \& Crespí, HVR 11420; Vila Nova de Foz Côa, estação de Castelo Melhor, 29TPF65, 2.5.1996, Sequeira \& Santos, HVR 7868.

PO, TRÁS-OS-MONTES: Vila Real, Sabrosa, 29TPF06, 4.5.1988, Coelho, HVR 4247; Vimioso, Algoso, no castelo, 29TQG00, 23.4.1992, C. Aguiar 1190, BRESA 1662; margem direita do Douro, à entrada da Régua, borda da estrada Régua-Caldas do Moledo, junto à lixeira, 150-200 m, 29TPF 05, 4.5.1993, Sequeira 590A, HVR 4195.

SPA, SALAMANCA: Saucelle, Salto de Saucelle, riberas del río Duero, $190 \mathrm{~m}$, 29TPF8446, 15.5.2003, Amich, SALA 108457.

En la reciente revisión de la sección Leptocrambe DC. (Prina, 2000) este autor separa las poblaciones del taxon linneano distribuidas en el mediterráneo occidental y norte de África como subsp. glabrata, mientras que las del mediterráneo centro-oriental corresponderían a la subsp. hispanica. Las poblaciones estudiadas por nosotros en los territorios lusitano durienses del valle del Duero y alguno de sus afluentes presentan los ejemplares más o menos híspidos carácter de la subsp. hispanica-, si bien el artejo superior del fruto mide 3-5 mm -carácter de la subsp. glabrata-. Gómez Campo (1996) señala que en la Península coexisten las plantas con y sin indumento, y que son necesarios estudios adicionales, fundamentalmente cariológicos, para aclarar la situación; posteriormente, y empleando análisis de secuencias moleculares ITS FranciscoOrtega et al. (1999) ponen de manifiesto que la subsp. hispanica -tetraploide- está más cercana a otros táxones poliploides, como C. abyssinica, que a la planta de De Candolle. A pesar de la variabilidad del indumento, el tamaño de los frutos nos hace considerar las poblaciones de la cuenca del Duero como subsp. glabrata, de acuerdo con Prina (2000).

Trigonella polyceratia L. subsp. amandiana (Samp.) Amich \& J. Sánchez

PO, TRÁS-OS-MONTES: Carrazeda de Ansiães, Lousa, em frente à estação de Freixo de Numão, ruderal, 29TPF44, 5.4.1993, C. Aguiar 1665, BRESA 2230; Freixo de Espada à Cinta, Ligares, Quinta da Barca, em frente a Barca D' 
Tabla 1

Sileno duriensis-Aphyllanthetum monspeliensis Amado, Honrado \& Aguiar in Aguiar et al. 2003 (Sideritido Salvion, Rosmarinetalia, Rosmarinetea)

\begin{tabular}{lcccccc}
\hline Inventario $\mathrm{n}^{\mathrm{o}}$ & 1 & 2 & 3 & 4 & 5 & 6 \\
Altitud $(\mathrm{m})$ & 135 & 330 & 500 & 325 & 120 & 250 \\
Cobertura $(\%)$ & 90 & 80 & 55 & 70 & 60 & 50 \\
Area $\left(\mathrm{m}^{2}\right)$ & 50 & 50 & 75 & 75 & 50 & 50 \\
Exposición & $\mathrm{W}$ & $\mathrm{NW}$ & $\mathrm{W}$ & $\mathrm{NW}$ & $\mathrm{NW}$ & $\mathrm{NE}$ \\
Inclinación $\left({ }^{\circ}\right)$ & - & 10 & 10 & 10 & - & 10
\end{tabular}

\section{Características}

Coronilla minima subsp. lotoides

Aphyllanthes monspeliensis

Thymus zygis

Avenula bromoides

Galium glaucum subsp. australe

Silene boryi subsp. duriensis

Petrorhagia saxifraga

Globularia vulgaris

Sedum sediforme

Scorzonera hispanica subsp. crispatula

Biscutella valentina

Dorycnium pentaphyllum

Hippocrepis comosa

Sideritis hirsuta subsp. danielii

Centaurea melitensis

Dactylis hispanica

$\begin{array}{llllll}3 & 3 & 2 & 2 & 2 & 1 \\ 3 & 2 & 2 & + & 2 & . \\ 2 & 2 & 2 & 2 & 1 & 1 \\ 1 & 1 & 1 & 2 & . & 2 \\ 1 & 1 & 1 & 1 & 1 & 2 \\ + & 1 & + & . & . & 1 \\ 2 & 2 & . & 2 & 1 & 1 \\ + & 2 & 1 & . & . & 2 \\ 2 & + & . & 1 & 1 & 1 \\ 1 & 1 & . & 1 & . & 2 \\ + & 1 & . & 1 & . & 1 \\ 1 & 1 & 2 & . & . & 1 \\ 2 & 1 & . & . & 1 & . \\ 1 & 1 & . & . & 1 & 1 \\ + & + & 1 & 2 & 1 & 1\end{array}$

\section{Compañeras}

Arabis planisiliqua

Prunus mahaleb

Melilotus spicata

Pistacia terebinthus

Phagnalon saxatile

Campanula erinus

Clematis campaniflora

Asparagus acutifolius

Daphne gnidium

Genista hystrix

Osyris alba

Jasminum fruticans

Sedum acre

Bituminaria bituminosa

Brachypodium dystachion

Lathyrus clymenum

Polygala microphylla

Mercurialis tomentosa

Peucedanum officinale

$\begin{array}{llllll}+ & + & 1 & 1 & 1 & . \\ + & 1 & 1 & 1 & 1 & 1 \\ 1 & 1 & + & 1 & 1 & 1 \\ 1 & + & 1 & 1 & + & + \\ 1 & 1 & 1 & 1 & 1 & 1 \\ 1 & 1 & . & 1 & 1 & 1 \\ 1 & 1 & 2 & . & 1 & 1 \\ + & . & 1 & 1 & 1 & 1 \\ 1 & . & + & 1 & . & . \\ 1 & . & 1 & . & 1 & . \\ 1 & . & . & 1 & 1 & . \\ 1 & . & 1 & . & 1 & 1 \\ 1 & 1 & . & . & . & . \\ 1 & + & . & . & + & + \\ 1 & + & . & . & 1 & . \\ . & 1 & 1 & . & . & . \\ + & 1 & . & . & . & . \\ 1 & 1 & . & . & . & 1 \\ 1 & 1 & . & . & . & \end{array}$

Localidades. 1: Salamanca, Salto de Saucelle, 29TPF8446. 2: Salamanca, Salto de Villarino, Casas del Rosquillero, 29TQF6871. 3: Zamora, Castro de Alcañices, proximidades del Embalse de Castro, 29TQG3506. 4: Salamanca, Villarino, 29TQF6871. 5: Salamanca, La Fregeneda, Muelle de Vegaterrón, 29TPF7444. 6: Salamanca, Salto de Aldeadávila, 29TPF9463. 
Alva, 29TPF8945, 23.4.1994, C. Aguiar 2400, BRESA 2869; Vila Nova de Foz Coa, Pocinho, 115 m, 29TPF5857, 25.4.1995, Amich et al. s.n. HVR; Río Douro, a jusante da foz do río Sabor, na ilha, $425 \mathrm{~m}, 29 \mathrm{TPF} 5857,30.4 .1995, M$. Sequeira 907, HVR 7333.

Los monógrafos del género en Flora iberica (Hedge \& Sales, 2000) consideran uno de los principales caracteres diagnósticos de este taxon -pedúnculos de las inflorescencias de 3-4 cm (Amich \& Sánchez, 1983)- como "variaciones de carácter local" y no lo tratan como diferente de la planta linneana. No deja de parecernos sorprendente dicha opinión si tenemos en cuenta que dichos autores utilizan el carácter del tamaño de los pedúnculos de la inflorescencia como el primero discriminatorio en la clave del género. En las poblaciones estudiadas por nosotros el tamaño de los pedúnculos -tanto de la inflorescencia como fructíferos- es siempre un carácter constante, no apareciendo nunca ejemplares con las inflorescencias sésiles. Sirjaev (1931) indica que se trata de una notable variedad y del único taxon dentro del complejo específico de $T$. polyceratia con legumbres no sésiles.

Las plantas con este carácter presentan un área de distribución propia a lo largo del río Duero en España (Zamora y Salamanca) y Portugal (Trás-os-Montes y Douro Litoral), es decir, en el sector biogeográfico Lusitano Duriense, así como una particular ecología, integrándose en las comunidades de pastizales terofíticos correspondientes a la alianza Corynephoro articulati-Malcomion patulae; por ello creemos claramente justificada su consideración con este rango.

\section{Cytinus ruber Fourr. ex Fritsch}

PO, TRÁS-OS-MONTES: São João da Pesqueira, Vale de Figueira, riberas del río Duero, $300 \mathrm{~m}, 29 \mathrm{TPF} 3755,21.4 .2001$, Amich \& Bernardos, Diapositiva $\mathrm{n}^{\circ} .4122$.

Esta endoparásita no se conocía de Trás-osMontes (Villar, 1997). La citamos ahora en el límite entre esta provincia y la de la Beira Alta, en las riberas del río Duero, viviendo sobre jaras blancas (Cistus albidus) en el seno de los alcornocales termófilos con oxicedros característicos de estos territorios lusitano durienses (Junipero lagunae-Quercetum suberis).
Pinguicula lusitanica L.

SPA, CÁCERES: Ríomalo de Abajo, riberas del río Ladrillar, 405 m, 29TQE4577, 30.5.2003, Amich \& Bernardos, SALA 108453.

SPA, SALAMANCA: La Herguijuela de la Sierra, arroyo de La Juria, 555 m, 29TQE4579, 17.5.2003, Amich \& Bernardos, SALA 108451; La Herguijuela de la Sierra, La Rebollosa, E1 Carcavón, 425 m, 29TQE4577, 30.5.2003, Amich \& Bernardos, SALA 108449; La Herguijuela de la Sierra, Valle de Belén, 820 m, 29TQE4580, 30.5.2003, Amich \& Bernardos, SALA 108452; La Herguijuela de la Sierra, La Rebollosa, arroyo de Lomo Pinto, 700 m, 29TQE4479, Bernardos et al., SALA 108450.

Se trata de un taxon de distribución típicamente atlántica, que en la Península Ibérica se presenta sobre todo en la mitad occidental (Blanca et al., 1999) y que recientemente Sánchez Rodríguez et al. (2000) indicaron como novedad para el Sistema Central en una localidad salmantina. Aportamos ahora nuevas localidades de esta particular insectívora, tanto en la provincia de Salamanca -aunque en este caso en el sector Toledano Tagano- como en la vecina de Cáceres, dónde sólo se conocía la antigua localización de Ladero en la Sierra de Guadalupe (MAF 81711). En todas ellas P. lusitanica se presenta localmente copiosa, y creemos que puede estar aún más ampliamente extendida a lo largo de estos territorios de la cuenca media del río Alagón.

La posición sintaxonómica de las comunidades en que habita no es clara, al igual que ocurre con otras similares, pioneras de aguas oligótrofas en las depresiones que aparecen en el seno de las turberas o en sus claros. Aunque en ocasiones aparece viviendo en comunidades que podrían ser referibles a Eleocharito multicaulisRynchosporetum albae (Rynchosporion albae, Scheuchzerietalia palustris) [Tabla 2, inv. 1; Sánchez Rodríguez et al., 2000], fitocenosis supramediterráneas Carpetano-Leonesas, en la mayor parte de las ocasiones [Tabla 2, invs. 2-5] pensamos que las fitocenosis en que vive cabría adscribirlas a la alianza Anagallido tenellaeJuncion bulbosi (Caricetalia nigrae).

Dentro de esta alianza se engloban tres asociaciones (Rivas-Martínez et al., 2001): dos de distribución preferentemente Cántabro- 
Tabla 2

Eleocharito multicaulis-Rynchosporetum albae C. Valle \& F. Navarro ex Rivas-Martínez 2002 [inv. $\mathrm{n}^{\circ} 1$ ]

(Rynchosporion albae, Scheuchzerietalia palustris, Scheuchzerio-Caricetea fuscae)

Sibthorpio europeae-Pinguiculetum lusitanicae

Ladero \& A.Velasco in A.Velasco 1980 [invs. 2-5]

(Anagallido-Juncion bulbosi, Caricetalia nigrae, Scheuchzerio-Caricetea fuscae).

Inventario $\mathrm{n}^{\mathrm{o}}$

Altitud (m)

Cobertura (\%)

Area $\left(\mathrm{m}^{2}\right)$

Exposición

Inclinación $\left({ }^{\circ}\right)$

12

$820 \quad 555$

$30 \quad 75$

21

- $\quad$ SE

$\begin{array}{ll}- & 10\end{array}$

3
425
80
0,75
$\mathrm{~S}$
10

4

405

85

0,75

$\mathrm{N}$

10
5

700

60

2

SE

10

\section{Características}

Pinguicula lusitanica

Drosera rotundifolia

Lycopodiella inundata

Juncus bulbosus

Anagallis tenella

Sphagnum denticulatum

Eleocharis multicaulis

Pedicularis lusitanica

Juncus pygmaeus

Carex binervis

Cicendia filiformis

Radiola linoides

Illecebrum verticillatum

Isoetes hystrix

Wahlenbergia hederacea

Centaurium maritimum

Juncus buffonius

Spiranthes aestivalis

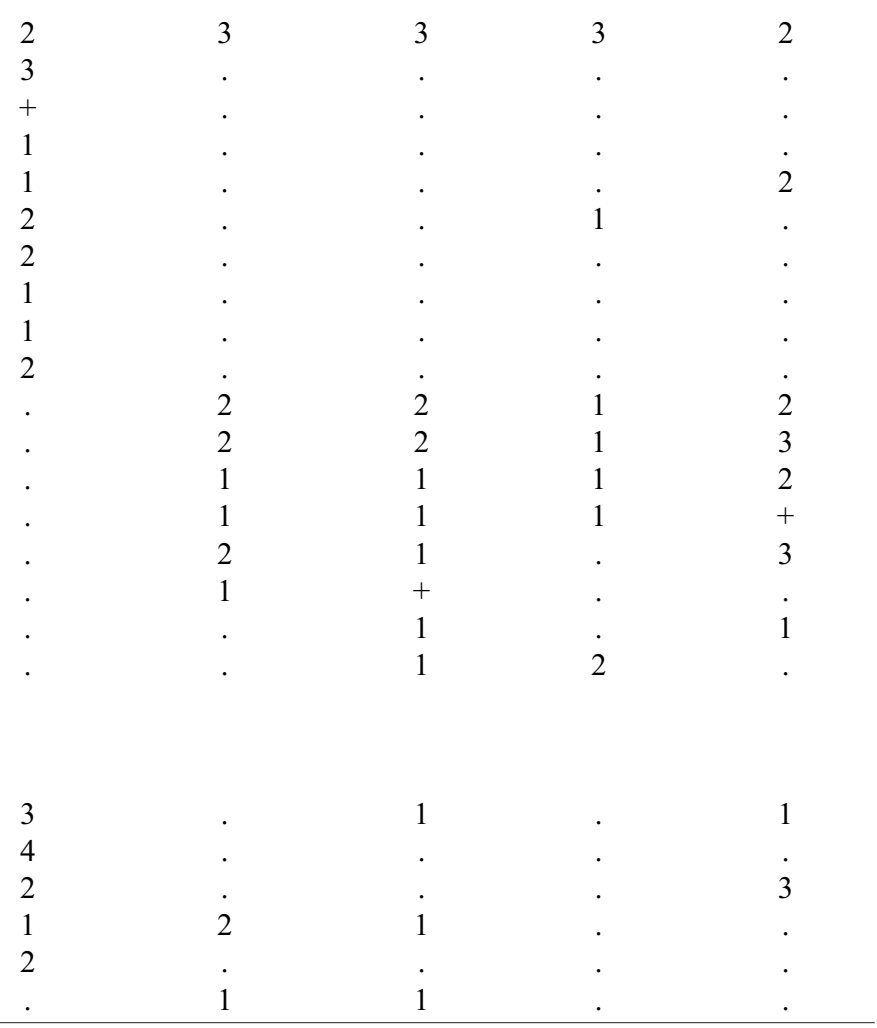

Localidades. 1: Salamanca, La Herguijuela de la Sierra, Valle de Belén, 29TQE4580. 2: Salamanca, La Herguijuela de la Sierra, arroyo de La Juria, 29TQE4579. 3: Salamanca, La Rebollosa, El Carcavón, 29TQE4577. 4: Cáceres, Ríomalo de Abajo, márgenes del río Ladrillar, 29TQE4577. 5: Salamanca, La Rebollosa, arroyo de Lomo Pinto, 29TQE4479. 
Atlántica, Anagallido tenellae-Juncetum bulbosi y Arnicetum atlanticae, y la tercera LusoExtremadurense, Sibthorpio europaeaePinguiculetum lusitanicae.

La ausencia total -o casi- en nuestras comunidades de elementos atlánticos como Arnica montana subsp. atlantica, Anagallis tenella o Scutellaria minor, así como su ecología, en márgenes y pequeños taludes de arroyos montanos, pensamos que hace aconsejable llevar las mismas a Sibthorpio-Pinguiculetum. Este argumento se ve reforzado además desde un punto de vista biogeográfico, dado que los territorios en los que se presentan estas fitocenosis pertenecen a la subprovincia Luso-Extrema-durense.

Sideritis hirsuta subsp. danielii (Rivera \& Obón) Bernardos, Aguiar \& Amich, comb. \& stat. nov.

Basiónimo: Sideritis danielii Rivera \& Obón in A taxonomic revision of the section Sideritis (genus Sideritis) (Labiatae), Phaner. Monog. Tomus XXI: 413 (1994)

PO, TRÁS-OS-MONTES: Freixo de Espada à Cinta, Poiares, em frente a Barca d'Alva, olival, 29TPF84, 29.3.1994, C. Aguiar 2337, BRESA 4274; Bemposta, barragem de Bemposta, $360 \mathrm{~m}$, 29TQF1174, 15.5.2003, Amich \& Amado, Diapositiva $\mathrm{n}^{\circ} 5297$.

SPA, SALAMANCA: Villarino de Los Aires, Salto de Villarino, riberas del río Duero, 345 m, 29TQF6871, 15.5.2003, Amich, SALA 108456; Sotoserrano, riberas del río Francia, 485 m, 29TQE5380, 12.7.2003, Amich \& Bernardos, SALA 108454.

Llevamos a este taxon los materiales colectados en diversas localidades de la cuenca del río Duero, así como en la cuenca del Alagón, caracterizados por sus hojas algo más anchas y la pelosidad típicamente holótrica y muy frecuentemente homótrica. En base a estos caracteres, consideramos más adecuado el rango subespecífico, que ahora proponemos. Algunas antiguas recolecciones de este taxon en el sector lusitano duriense (Arribes del Duero) también corresponden a este taxon (Villarino de los Aires, 13.5.1976, J. Sánchez, SALA 17733; La Fregeneda, 2.6.1978, Amich, SALA 16268).

Representa novedad para las provincias de Salamanca y Trás-os-Montes. En esta última fue recientemente señalado S. bubani Font Quer (Amado et al. in Aguiar et al., 2003), cita que corresponde al taxon que ahora nos ocupa; la planta de Font Quer sólo es conocida del NE español (Obón \& Rivera, 1994).

$S$. danielii se integra en las fitocenosis referibles al sinendemismo lusitano duriense Sileno duriensis-Aphyllanthetum monspeliensis (tab. 1), que anteriormente comentamos con detalle.

Antirrhinum lopesianum Rothm.

PO, TRÁS-OS-MONTES: Bragança, Grijó de Parada, Cabeço das Freiras, junto ao rio Sabor, afloramentos calcários em escarpas rochosas de xisto, 490 m, 29TPG9225, 1.6.1992, C. Aguiar 1390, BRESA 1651; Vimioso, Carção, junto à ponte sobre o rio Maçãs, afloramentos calcários em escarpas rochosas de xisto, $455 \mathrm{~m}$, 29TQG0309, 14.5.1993, C. Aguiar 2466, BRESA 2466; Bragança, Grijó de Parada, escarpas sobranceiras ao rio Sabor, xisto com intercalações calcárias, 500 m, 29TPG9225, 25.5.1996, C. Aguiar 3291, BRESA 3927.

SPA, SALAMANCA: Aldeadávila de La Ribera, riberas del río Duero, 325 m, 29TPF9969, 21.5.2003, Amado et al., SALA 108448.

SPA, ZAMORA: Pinilla de Fermoselle, riberas del río Duero, $400 \mathrm{~m}, 29 \mathrm{QF} 1884$, 22.5.2003, Amado et al., Diapositiva no 5304.

Señalado como novedad para la Flora española por Amich et al. (1989) en Las Arribes del Duero salmantinas, recientemente se dió a conocer una nueva localidad en la provincia de Zamora (Bernados et al., 2003). Aportamos ahora dos nuevas localidades, respectivamente en las provincias de Salamanca (Aldeadávila de La Ribera) y Zamora (Pinilla de Fermoselle). Ambas poblaciones cuentan con un número importante de individuos (alrededor de 200) y se encuentran en buen estado de conservación, probablemente como consecuencia de la considerable dificultad de acceso a los emplazamientos en que vive. En cualquier caso, y a pesar de estas dos nuevas poblaciones que ahora señalamos, se trata de una planta fuertemente estenoica, considerada en la categoría "en peligro" (EN) (González-Talaván et. al. in Bañares et al., 2003) que limita su área de distribución a 4 localidades españolas 
disyuntas a lo largo del río Duero, entre los embalses de Castro y Aldeadávila, y otras tantas en la cuenca medio-alta del río Sabor, en la provincia portuguesa de Trás-os-Montes.

Se integra en comunidades de Rumici indurati-Dianthion lusitani, en concreto en la asociación Phagnalo-Antirrhinetum lopesianii, recientemente descrita (Bernardos et al., 2004) para estos territorios lusitano durienses.

Scrophularia valdesii Ortega-Olivencia \& Devesa

PO, TRÁS-OS-MONTES: Freixo, Ventozelo, riberas del Douro, $350 \mathrm{~m}$, 29TQF0169, 22.5.2003, Amado et al., SALA 108455.

SPA, SALAMANCA: Saucelle, Salto de Saucelle, 175 m, 29TPF8446, 27.6.1996, Amich \& Hernández-Toro, SALA 88687; Corporario, El Rostro, riberas del río Duero, 340 m, 29TQF0168, 20.5.2002, Amich \& Bernardos, Diapositiva ${ }^{\circ}$. 4657.

SPA, ZAMORA: Castro de Alcañices, Embalse de Castro, $545 \mathrm{~m}, 29 \mathrm{TQG} 3506$, 27.5.2002, Bernardos \& González-Talaván, Diapositiva ${ }^{\circ} .4667$.

La localidad portuguesa representa la segunda cita de esta especie en el país, tras la reciente señalización de Marcos et al. (2004); se trata de una localidad intermedia entre las señaladas por estos autores en los concelhos de Freixo y Mirando do Douro.

Las localidades españolas, salmantinas y zamoranas, amplían el área conocida de este relevante endemismo lusitano duriense (OrtegaOlivencia \& Devesa, 1991, 1993; OrtegaOlivencia \& Rodríguez-Liaño, 2002).

Considerada como "en peligro crítico" (CR) en la Lista Roja de la Flora Vascular Española (VV.AA., 2000), estudios más detallados sobre el taxon aconsejan una categoría de "vulnerable" (VU) (González-Talaván et al. in Bañares et al., 2003).

Se integra tanto en comunidades pertenecientes al sinendemismo lusitano duriense Phagnalo-Antirrhinetum lopesianii (Bernardos et al., 2004) como en los emplazamientos escionitrófilos que aparecen en la base de esos roquedos, en formaciones referibles a ChelidonioSmyrnietum olosatri.

\section{SINTAXONOMÍA DE LAS COMUNIDADES CITADAS EN EL TEXTO}

14. SCHEUCHZERIO-CARICETEA FUSCAE Tüxen 1937

14a. Scheuchzerietalia palustris Nordhagen 1936

14.1. Rhynchosporion albae Koch 1926

14.1.3. Eleocharito multicaulisRhynchosporetum albae C. Valle \& F. Navarro ex Rivas-Martínez 2002

14b. Caricetalia fuscae Koch $1926 \mathrm{em}$. Br.-B1. 1949

14.3. Anagallido tenellae-Juncion bulbosi Br.B1. 1967

14.3.1. Anagallido tenellae-Junceum bulbosi Br.B1. 1967

14.3.2. Arnicetum atlanticae Bellot 1968

14.3.3. Sibthorpio europeae-Pinguiculetum lusitanicae Ladero \& A. Velasco in A. Velasco 1980

32. PHAGNALO-RUMICETEA INDURATI (Rivas Goday \& Esteve 1972) RivasMartínez, Izco \& Costa 1973

32a. Phagnalo saxatilis-Rumicetalia indurati Rivas Goday \& Esteve 1972

32.3. Rumici indurati-Dianthion lusitani RivasMartínez, Izco \& Costa 1973 ex Fuente 1986

32.3.15. Phagnalo saxatilis-Antirrhinetum lopesianii Bernardos, Crespí, Aguiar, F.J. Fernández \& Amich 2004

40. GALIO-URTICETEA Passarge \& Kopeck 1969

40a. Galio aparines-Alliarietalia petiolatae Görs \& Müller 1969

40.2. Galio-Alliarion petiolatae Oberdorfer \& Lohmeyer in Oberdorfer, Görs, Korneck, Lohmeyer, Müller, Philippi \& Seibert 1967

40.2.12. Chelidonio majoris-Smyrnietum olosatri Amigo \& Romero 1997

50. HELIANTHEMETEA GUTTATI (Br.-B1. in Br.-B1., Roussine \& Nègre 1952) Rivas Goday \& Rivas-Martínez 1963 em. RivasMartínez 1978

50b. Malcolmietalia Rivas Goday 1958

50.5. Corynephoro articulati-Malcomion patulae Rivas Goday 1958

64. ROSMARINETEA OFFICINALIS Rivas- 
Martínez, T.E. Díaz, F. Prieto, Loidi \& Penas 2002

64a. Rosmarinetalia officinalis Br.-B1. ex Moliner 1934

64.5. Sideritido incanae-Salvion lavandulifoliae (Rivas Goday \& Rivas-Martínez 1969) Izo \& A. Molina 1989

64.5a Xero-Aphyllantenion Rivas Goday \& Rivas-Martínez 1969 em. Izco \& A. Molina 1989

64.5.8. Sileno duriensis-Aphyllanthetum monspeliensis Amado, Honrado \& Aguiar 2003

75. QUERCETEA ILICIS Br.-B1. ex A. \& O. Bolòs 1950

75a. Quercetalia ilicis Br.-B1. ex Moliner 1934 em. Rivas-Martínez 1975

75.2. Quercion broteroi Br.-B1., P. Silva \& Rozeira 1956 em. Rivas-Martínez 1975 corr. Ladero 1974

75.2.4.Junipero lagunae-Quercetum suberis Rivas-Martínez, Aguiar, Cantó \& Ladero 2002

AGRADECIMIENTOS. Agradecemos a Jesús Leguía la amable indicación de algunas localidades. Este trabajo ha sido realizado gracias a la ayuda de la Comunidad Autónoma de Castilla y León (SA037/02).

\section{BIBLIOGRAFÍA}

Aguiar, C., J.C. COSTA, J. CAPELO, A. AMADO, J. HONRADO, M.D. ESPÍRITO SANTO y M. LOUSÃ -2003- Aditamentos à vegetação de Portugal continental. Nota 34 en Notas do Herbario da Estação Florestal Nacional (LISFA). Fasc. XVII. Silva Lusit. 11 (1): 101-111.

ÁlVAREZ, I., A. HERRERO y B. VEGA -1996Aportación 86. Cleome violacea L. En: Rubio Sánchez, A. (ed.) Cartografía corológica ibérica. Bot. Complutensis 20: 170-173.

ÁlVAREZ MAESTRE, P.-A. y J.M. SANTOS ESTEBAN -1996- Mapa 738. Cleome violacea L. En: Fernández Casas, J. (ed.) Asientos para un atlas corológico de la flora occidental, 24. Fontqueria 44: 197-200.

AMADO, A. y C. AGUIAR -2004- O género
Fumana (Dunal) Spach em Trás-os-Montes. De Novarum Flora Lusitani Commentarii - I. Nota 10 en Notas do Herbário da Estação Florestal Nacional (LISFA). Fasc. XVIII. Silva Lusit. 11 (2): 232-233.

AMICH, F. -1980- Datos acerca de la flora salmantina. II. Trab. Dep. Bot. Salamanca 9:5-18.

AMICH, F. \& J. SÁNCHEZ SÁNCHEZ -1983Acerca de las subespecies de Trigonella polyceratia L. Stud. Bot. Univ. Salamanca 2: 129-132.

AMICH, F., J.A. SÁNCHEZ RODRÍGUEZ, F. GALLEGO y M.A. SÁNCHEZ ANTA -1989Antirrhinum lopesianum Rothm., novedad para la flora española. Bol. Soc. Brot., Sér. 2, 52: 231-237.

BERNARDOS, S., C. AGUIAR y A. GONZÁLEZ-TALAVÁN -2003- Segunda localidad española de Antirrhinum lopesianum Rothm. (Scrophulariaceae). Anales Jard. Bot. Madrid 60: 228-229.

BERNARDOS, S., A.L. CRESPÍ, C. AGUIAR, J. FERNÁNDEZ y F. AMICH -2004- The plant communities of the Rumici induratiDianthion lusitani alliance in the Lusitan Duriensean biogeographical sector (NE Portugal and CW Spain). Acta Bot. Gallica 151: en prensa.

BLANCA, G., M. RUIZ-TEJÓN y R. ZAMORA -1999- Taxonomic revision of the genus Pinguicula L. in the Iberian Peninsula. Folia Geobot. 34: 337-361.

CASTROVIEJO, S. et al. (eds.) -1986-1997 y 2003- Flora iberica. Vols. I-V, VIII y X. Madrid: Real Jardín Botánico, C.S.I.C.

CRESPÍ, A.L., J. GUIMARAES, S. BERNARDOS, C.P. FERNÁNDEZ, A. CASTRO, A. PEREIRA, T. MALFEITO y F. AMICH -2003- Contribución al conocimiento de la diversidad específica del género Medicago L. para el Norte de Portugal. Bot. Complutensis 27: 105-117.

FRANCISCO-ORTEGA, J., J. FUERTESAGUILAR, C. GÓMEZ-CAMPO, A. SANTOS-GUERRA y R.K. JANSEN -1999Internal transcribed spacer sequence phylogeny of Crambe L. (Brassicaceae): Molecular data reveal two Old World disjunctions. Mol. Phylogenet. Evol. 11:361- 
380.

GÓMEZ CAMPO, C. -1996- Crambe L. En: Castroviejo, C., C. Aedo, C. Gómez Campo, M. Laínz, P. Montserrat, R. Morales, F. Muñoz Garmendia, G. Nieto Feliner, E. Rico, S. Talavera y L. Villar (eds.) Flora iberica 4: 429-431. Madrid: Real Jardín Botánico, C.S.I.C.

GONZÁLEZ-TALAVÁN, A., S. BERNARDOS y F. AMICH -2003-Antirrhinum lopesianum Rothm. En: Bañares, A., G. Blanca, J. Güemes, J.L. Moreno y S. Ortiz (eds.) Atlas y Libro Rojo de la Flora Vascular Amenazada de España. Madrid: Dir. Gral. Conserv. Nat., 584-585.

GONZÁLEZ-TALAVÁN, A., M. SANTOS VICENTE, S. BERNARDOS, P. BARIEGO y F. AMICH -2003- Scrophularia valdesii Ortega Olivencia \& Devesa. En: Bañares, A., G. Blanca, J. Güemes, J.L. Moreno y S. Ortiz (eds.) Atlas y Libro Rojo de la Flora Vascular Amenazada de España. Madrid: Dir. Gral. Conserv. Nat., 908.

HEDGE, I.C. y F. SALES -2000- Trigonella L. En: Talavera, S., C. Aedo, S. Castroviejo, A. Herrero, C. Romero Zarco, F.J. Salgueiro y M. Velayos (eds.) Flora iberica 7: 731-741. Madrid: Real Jardín Botánico, C.S.I.C.

MARCOS, N., A. AMADO y C. AGUIAR -2004Scrophularia valdesii Ortega Olivencia \& Devesa - confirmada a presença em Portugal de mais um endemismo lusitano-duriense. De Novarum Flora Lusitana Commentarii - I. Nota 12 en Notas do Herbário da Estação Florestal Nacional (LISFA). Fasc. XVIII. Silva Lusit. 11 (2): 233-234.

MUÑOZ GARMENDIA, F. y C. NAVARRO (eds.) -1998-Flora iberica. Vol. VI. Madrid: Real Jardín Botánico, C.S.I.C.

OBÓN DE CASTRO, C. y D. RIVERA NUÑEZ -1994- A taxonomic revision of the section Sideritis (genus Sideritis) (Labiatae). Phanerogamarum Monographiae Tomus XXI. Berlín: J. Cramer.

ORTEGA-OLIVENCIA, A. y J.A. DEVESA 1991- Dos nuevos táxones del género Scrophularia: S. viciosi y S. valdesii. Candollea 46: 111-118.

ORTEGA-OLIVENCIA, A. \& J.A. DEVESA 1993- Revisión del género Scrophularia
(Scrophulariaceae) en la Península Ibérica e Islas Baleares. Ruizia 11: 5-157.

ORTEGA-OLIVENCIA, A. \& T. RODRÍGUEZLIAÑO -2002- Novedades corológicas del género Scrophularia en España. Acta Bot. Malacitana 27: 269.

PAIVA, J. et al. (eds.) -2001- Flora iberica. Vol. XIV. Madrid: Real Jardín Botánico, C.S.I.C.

PRINA, A. -2000- A taxonomic revisión of Crambe, sect. Leptocrambe (Brassicaceae). Bot. J. Linn. Soc. 133: 509-524.

RICO, E. -1980-Aportaciones a la flora salmantina. Anales Jard. Bot. Madrid 36: 245255.

RIVAS-MARTÍNEZ, S., T.E. DÍAZ, F. FERNÁNDEZ-GONZÁLEZ, J. IZCO, J. LOIDI, M. LOUSÃ y A. PENAS -2002Vascular plant communities of Spain and Portugal. Itinera Geobot. 15: 1-922.

RIVAS-MARTÍNEZ, S., F. FERNÁNDEZ GONZÁlEZ, J. LOIDI, M. LOUSÃ y A. PENAS -2001- Syntaxonomical checklist of vascular plant communities of Spain and Portugal to association level. Itinera Geobot. 14: 5-341.

RIVAS-MARTÍNEZ, S., J. LOIDI, M. COSTA, T.E. DÍAZ, y A. PENAS -1999- Iter Ibericum A.D. MIM. Itinera Geobot. 13: 5-347.

SÁNCHEZ RODRÍGUEZ, J.A., J. LEGUÍA SEBASTIÁN y M.A. MARTÍN MARCOS 2000- Pinguicula lusitanica L. (Lentibulariaceae), novedad para el Sistema Central. Anales Jard. Bot. Madrid 58: 196197.

SIRJAEV, G. -1931- Generis Trigonella L. Revisio Critica IV. Spisy Prír. Fak. Masarykovy Univ. 136: 1-33.

TALAVERA, S. -1990- Silene L. En: Castroviejo, S., M. Laínz, G. López González, P. Montserrat, F. Muñoz Garmendia, J. Paiva y L. Villar (eds.) Flora iberica 2: 313-406. Madrid: Real Jardín Botánico, C.S.I.C.

TALAVERA, S. y E. DOMÍNGUEZ -2000Hippocrepis L. En: Talavera, S., C. Aedo, S. Castroviejo, A. Herrero, C. Romero Zarco, F.J. Salgueiro y M. Velayos (eds.) Flora iberica 7: 897-935. Madrid: Real Jardín Botánico, C.S.I.C.

TALAVERA, S. et al. (eds.) -1999-2000- Flora iberica. Vol VII ( 1 y 2). Madrid: Real Jardín 
Botánico, C.S.I.C.

TUTIN, T. G. et al. (eds.) -1964/1980- Flora Europaea. Vols. 1-5. Cambridge: Cambridge University Press.

VILLAR, L. -1997-Cytinus L. En: Castroviejo, S., C. Aedo, C. Benedí, M. Laínz, F. Muñoz Garmendia, G. Nieto Feliner y J. Paiva (eds.) Flora iberica 8: 170-174. Madrid: Real Jardín Botánico, C.S.I.C.

VV.AA. -2000- Lista Roja de la Flora Vascular Española (valoración según categorías UICN). Conserv. Veg. 6: 11-38.
Aceptado para su publicación en junio de 2004

Dirección de los autores. S. BERNARDOS y F. AMICH: Departamento de Botánica. Facultad de Biología. Universidad de Salamanca. E-37008 Salamanca. España; A. AMADO: Parque Natural do Douro Internacional. Rua Santa Marinha 4. 5200-241 Mogadouro. Portugal; C. AGUIAR: Escola Superior Agrária de Bragança. P-5300 Bragança. Portugal; A.L. CRESPÍ y A. CASTRO: Herbario e Jardim Botanico. Universidade de Trás-os-Montes e Alto Douro. P-5001-911 Vila Real. Portugal.

\section{DATOS SOBRE EL GÉNERO GENTIANA}

\section{Gustavo RENOBALES}

Data on the genus Gentiana.

Palabras clave. Gentianaceae, Gentiana angustifolia.

Key words. Gentianaceae, Gentiana angustifolia.

Gentiana angustifolia Vill. subsp. corbariensis (Braun-Blanq.) Renob. comb. nov.

Basión.: Gentiana clusii E.P. Perrier \& Songeon subsp. corbariensis Braun-Blanq. in Bull. Soc. Bot. France 84 (9-10): 671 (1938)

Recientemente publicamos algunas precisiones taxonómicas y nomenclaturales sobre ciertos táxones ibéricos de la tribu Gentianeae (Renobales in Anales J. Bot. Madrid 60: 461 - 469, 2003). Allí argumentábamos por una parte la conveniencia del tratamiento de Gentiana occidentalis Jak. como subespecie de G. angustifolia Vill., dadas las escasas diferencias morfológicas y fitoquímicas entre ambos [cf. Laínz in Bol. Inst. Estud. Asturianos 3: 175 (1961)]. Por otro lado, también expresábamos el parecer de que las poblaciones orientales de G. occidentalis Jak. (las que fueron llamadas G. clusii subsp. corbariensis Braun-Blanq.) no difieren significativamente de las occidentales de este mismo taxon, y no se justifica su tratamiento como subespecie distinta, idea que también han defendido otros autores precedentes [von Hagen \& Kadereit in Bot. Jahrb. Syst. 122 (3): 331 (2000)]. Sin embargo, pasamos por alto en esa ocasión que, en el rango subespecífico, el epíteto de Braun-Blanquet es prioritario. Por medio de esta nota enmendamos el anterior olvido, y expresamos nuestro agradecimiento al Dr. S. Talavera, que nos advirtió del error.

Aceptado para su publicación en septiembre de 2004

Dirección del autor. Dpto. Biología vegetal y Ecología. Facultad de Farmacia. Universidad del País Vasco UPV / EHU. Apdo. 450. 01080 Vitoria. gvprescg@vc.ehu.es 